•研究报告・

\title{
基于MaxEnt模型预测白唇鹿的潜在分布区
}

\author{
崔绍朋1,2,3\# 罗 晓 $1,2 \#$ 李春旺 ${ }^{1,2}$ 胡慧建 ${ }^{4}$ 蒋志刚 $1,2^{*}$ \\ 1 (中国科学院动物研究所动物生态与保护生物学重点实验室, 北京 100101) \\ 2 (中国科学院大学, 北京 100049) \\ 3 (山西农业大学林学院, 山西太谷 030801) \\ 4 (广东省生物资源应用研究所, 广州 510260)
}

\begin{abstract}
摘要: 研究物种分布格局对制定有效保护措施至关重要。本研究从文献记录和实地调查中获取 97 个分布点, 篮选 出7个重要环境变量, 采用最大熵(MaxEnt)模型分析了中国青藏高原特有种白唇鹿(Przewalskium albirostris)的潜 在分布区域。结果显示: 白唇鹿潜在分布区主要位于青藏高原东部, 适宜生境呈现出以西藏、青海和四川三省交 界地带为核心向四周扩散的模式。刀切法(Jackknife)分析表明, 地形、气温季节变化和年降水量是决定白唇鹿分布 的重要因素, 人类影响变量贡献相对较小。白唇鹿种群现状尚不清晰，建议对该物种进行全面深入调查。
\end{abstract}

关键词：最大熵模型; 白唇鹿; 青藏高原; 潜在分布区

\section{Predicting the potential distribution of white-lipped deer using the Max- Ent model}

\author{
Shaopeng Cui ${ }^{1,2,3 \#}$, Xiao Luo ${ }^{1,2 \#}$, Chunwang $\mathrm{Li}^{1,2}$, Huijian $\mathrm{Hu}^{4}$, Zhigang Jiang ${ }^{1,2^{*}}$ \\ 1 Key Laboratory of Animal Ecology and Conservation Biology, Institute of Zoology, Chinese Academy of Sciences, Bei- \\ jing 100101 \\ 2 University of Chinese Academy of Sciences, Beijing 100049 \\ 3 College of Forestry, Shanxi Agricultural University, Taigu, Shanxi 030801 \\ 4 Guangdong Institute of Applied Biological Resources, Guangzhou 510260
}

\begin{abstract}
Species distribution is critical for developing effective conservation measures. The potential geographic distribution of the white-lipped deer (Przewalskium albirostris), which is endemic to the QinghaiTibetan Plateau, was delineated using the Maximum Entropy (MaxEnt) model with 97 occurrence records and 7 environmental variables. The species occurrences were collected from literature and field investigations. Our results showed that the potential range of the white-lipped deer included the eastern part of the Qinghai-Tibetan Plateau and the potential habitat spread from one core region to neighboring regions among Tibet, Qinghai, and Sichuan provinces. The Jackknife test indicated that the topographic variable, temperature seasonality, and annual precipitation were the most important predictive factors for the model, while the human activity variable made a relatively small contribution. The current distribution and status of the white-lipped deer on the Qinghai-Tibetan Plateau is unclear and we suggest further research is needed on the species.
\end{abstract}

Key words: MaxEnt; white-lipped deer; Qinghai-Tibetan Plateau; potential distribution

白唇鹿(Przewalskium albirostris)是中国特有种 (吴家炎和王伟, 1999), 为国家I级重点保护野生动 物, 被IUCN红色名录列为易危(VU) (Harris, 2015), 中国脊椎动物红色名录判定为濒危 $(\mathrm{EN})$ (蒋志刚等,
2016)。白唇鹿分布范围仅限于青藏高原东部 3,500-5,100 $\mathrm{m}$ 的高海拔地区(Smith和解炎，2009; 蒋志刚等, 2015), 包括西藏东部、四川西部、青海 东部和甘肃西部(Ohtaishi \& Gao, 1990), 集中分布

收稿日期: 2017-03-15; 接受日期: 2017-11-16

基金项目：国家重大研发计划项目(2016YFC0503303)、国家自然科学基金(31572260,31372175)和科技基础性专项(2013FY110300)

\# 共同第一作者 Co-first authors

* 通讯作者 Author for correspondence. E-mail: jiangzg@ioz.ac.cn 
在祁连山以南至昆仑山、唐古拉山和横断山脉之间 的高原(吴家炎和王伟，1999; 国家林业局，2009; Leslie, 2010)。由于偷猎和栖息地减少, 白唇鹿在青 藏高原上的分布范围较历史时期大为缩减, 且呈岛 状分布(吴家炎和王伟, 1999)。20世纪90年代的资源 调查显示白唇鹿分布区逐年缩小, 在局部地区已十 分罕见或绝迹, 如青海的分布区比 20 世纪 80 年代中 期至少缩小了 $65 \%$ (国家林业局, 2009)。因此, 开展 白唇鹿分布的研究显得尤为迫切。

物种分布动态研究对物种的评估和保护至关 重要(Thorn et al, 2009), 但仅靠野外调查所获得的 数据难以反映物种的整体分布格局。近年来, 物种 分布模型(species distribution models, SDMs)在野生 动植物的潜在生境预测方面得到广泛应用( $\mathrm{Hu}$ et al, 2010; Yu et al, 2013; 吴庆明等, 2016), 其中最大熵 模型(maximum entropy approach, MaxEnt; Phillips et al, 2006)备受关注。该方法只需要依据物种分布点 数据建模, 在分布点相对较少的情况下也能获得较 准确的预测结果(Pearson et al, 2007; Phillips \& Dudík, 2008; Saupe et al, 2015), 在濒危物种生境分 布预测研究中被广泛采用。

为此, 本研究通过查阅数据库和历史文献中白 唇鹿的分布资料, 结合野外调查数据, 采用MaxEnt 模型预测其潜在分布区, 以期为该物种及其栖息地 的保护与恢复提供参考。

\section{1 研究方法}

\section{1 数据采集和处理}

本研究从两个途径搜集白唇鹿分布点数据: 一 是文献记录, 主要参考了《中国哺乳动物分布》(张 荣祖, 1997)、《中国白唇鹿》(吴家炎和王伟, 1999) 和其他相关研究文献(Schaller, 1998; Harris et al, 1999; Leslie, 2010), 利用ArcGIS 9.3 (ESRI, Redland, USA)软件对相关地图进行几何校正, 提取记 录点的经纬度, 不同文献的记录点相互补充验证, 共得到白唇鹿分布点76个; 二是我们2009-2015年 的野外实际调查记录, 共 21 个分布点, 主要位于西 藏工布江达和四川察青松多地区, 分布点经纬度数 据通过手持GPS采集, 定位精度约 $5 \mathrm{~m}$ 。最终共获得 97 个白唇鹿分布点, 之后将所有分布点的经纬度数 据在 Excel 表格中汇总, 保存为 CSV 格式用于 MaxEnt模型计算。

\section{2 模型的选择和使用}

本研究共选择4类环境变量(附录 1 和附录 2$):$ (1) 生物气候变量, 共 19 个, 从 WorldClim 数据库 (Version 1.4)中下载(Hijmans et al, 2005); (2)地形变 量, 包括海拔和坡度, 从USGS's Hydro- $1 \mathrm{~K}$ 数据库 获得(USGS, 2001); (3)生物学变量, 包括春夏秋冬 四个季节的归一化植被指数 (normalized difference vegetation index, NDVI), 该指数能反映地表植被的 分布状况; (4) 人类影响变量, 包括人类足迹指数 (human footprint influence, HFI; Wildlife Conservation Society (WCS) \& Center for International Earth Science Information Network (CIESIN), 2005)和放牧 强度(stocking rate)。人类足迹指数整合了人口居住 地、土地利用类型、道路网络、基础设施建设等基 本图层, 可以代表人为活动对白唇鹿及其生境的总 体干扰(Sanderson et al, 2002)。放牧强度用研究区域 内家畜密度表示, 数据来自联合国粮农组织(Food and Agriculture Organization)。我们利用ArcGIS软件 将上述 27 个变量的数据精度统一重采样为 30 ", 且 每个栅格只保留 1 个分布点。

首先对研究区域内的19个生物气候变量进行 主成分分析, 选择贡献率较高的前两个作为主成 分, 并得到每个变量的标准化特征向量(附录1), 然 后对 27 个初始环境变量进行成对Pearson相关性检 验(附录2)。若相关系数 $|r| \geq 0.75$, 我们认为变量间 存在相关性, 并保留特征向量值较大的变量。最终, 我们选择了 7 个环境变量预测白唇鹿潜在分布范围, 包括气温季节变化、年降水量、夏季NDVI指数、 海拔、坡度、人类足迹指数和放牧强度 $($ 表 1$)$ 。

选择MaxEnt 3.3.3k (Phillips et al, 2006)建立物 种分布模型, 设置随机检验百分比 (random test percentage) 为 $20 \%$, 重复数设为 10 , 重复运行类别 选择交叉验证(cross-validate), 其他参数默认设置。 以受试者工作特征曲线下面积 (area under the receiving operator curve, AUC)评价模型优劣; 采用刀切 法(Jackknife test)检验变量重要性。我们构建了10个 潜在分布模型, 计算不同模型的平均值整合为最 终预测结果。参照Liu等(2013), 选择最大训练敏感 度和特异度法(maximum training sensitivity plus specificity, MaxSS) 确定阈值, 将每个栅格的平均适 宜性(即出现概率, occurrence probability)进行二值 化处理, 最终得到白唇鹿在青藏高原的潜在分布区 
表1 预测白唇鹿潜在分布区时所用环境变量

Table 1 Environmental variables used to model the potential range of the white-lipped deer

\begin{tabular}{|c|c|c|}
\hline 编码 Code & 描述 Description & 来源 Source \\
\hline $\mathrm{Bio} 4$ & 气温季节变化 Temperature seasonality & WorldClim database Version 1.4 \\
\hline Bio12 & 年降水量 Annual precipitation & WorldClim database Version 1.4 \\
\hline NDVI_Summer & 夏季归一化植被指数 Normalized difference vegetation index in summer & Chinese Natural Resources Database \\
\hline Altitude & 海拔 Altitude & USGS's Hydro-1K dataset \\
\hline Slope & 坡度 Slope & USGS's Hydro-1K dataset \\
\hline HFI & 人类足迹指数 Human footprint influence & Last of the Wild Data Version 2 \\
\hline Stocking rate & 放牧强度 Stocking rate & Food and Agriculture Organization \\
\hline
\end{tabular}

域。最后，我们从IUCN Redlist下载白唇鹿现存分布 范围, 并将其与预测结果叠加。

\section{2 研究结果}

\section{1 模型评价及环境变量重要性}

MaxEnt模型结果显示, 10次交叉检验的平均训 练集AUC值为 $0.906 \pm 0.004($ mean $\pm \mathrm{SD})$, 模型的拟 合程度相对较高。刀切法分析了每个环境变量在预 测白唇鹿潜在分布区域时的重要性(图1), 结果表明 海拔对白唇鹿的分布影响最大, 温度季节变化、年 降水量以及夏季NDVI指数紧随其后但彼此之间差 别较小。总体而言, 地形变量和生物气候变量是影 响白唇鹿分布的最重要因素, 人类影响变量贡献相 对较小。

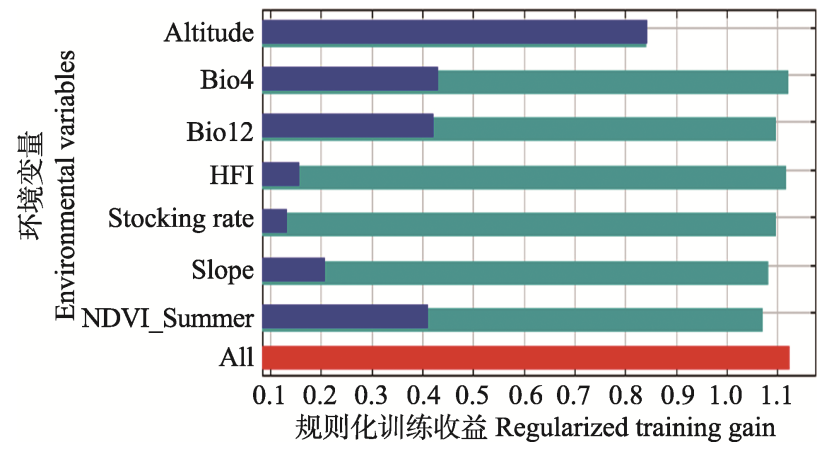

图1刀切法分析单个变量在建立分布模型时的重要性。对 每个变量而言, 蓝色表示只用该变量时的模型“收益”; 绿色 表示在移除该变量而使用其他变量建模时总体“收益”; 最 下方红色条带表示所有变量进入模型后的收益结果。环境变 量含义见表1。

Fig. 1 The Jackknife test for evaluating the relative importance of environmental variables in the development of the MaxEnt model. The blue bar shows the "total gain" achieved with the given predictor only. The green bar shows the "total gain" resulting from inclusion of all predictors except the given predictor. The bottom red bar shows the gain with all variables. For variable meanings, see Table 1.

\section{2 白唇鹿潜在分布区预测}

模型预测结果表明, 白唇鹿潜在生境分布在青 藏高原东部, 主要集中在西藏、青海和四川交界处, 如青海玉树和果洛藏族自治州、西藏昌都地区及四 川甘孜藏族自治州(图2)。总体上, 白唇鹿分布范围 呈现出以交界地带为核心向四周扩散的模式: 东至 四川盆地北部, 西侧分布于雅鲁藏布江和念青唐古 拉山附近高海拔地区, 北至祁连山脉, 南接横断山 脉地区。我们预测的白唇鹿潜在分布范围与其现存 范围大致相符, 但柴达木盆地南侧地区生境适宜性 偏低。在白唇鹿现存范围之外, 雅鲁藏布江和念青 唐古拉山附近地区明显适宜白唇鹿分布。另外, 青 海南山地区可能是白唇鹿南北种群交流的关键生 态廊道。

\section{3 讨论}

全球各个地区正发生不同程度的生物多样性 危机(蒋志刚和马克平, 2014), 而栖息地的破碎化和 丧失是导致生物多样性减少和物种灭绝的重要原 因(Fahrig，2003)。弄清受胁物种的潜在地理分布， 了解其适宜生境现状, 是有效开展保护工作的前提 (Pearce \& Boyce, 2006)。然而, 实际上许多物种的地 理分布数据很贫乏, 濒危物种更是如此。IUCN评估 结果认为白唇鹿种群密度低, 且呈零星分布, 种群 趋势未知(Harris, 2015)。本文预测的白唇鹿潜在活 动范围与现有研究结果相符(吴家炎和王伟, 1999; 国家林业局, 2009; Harris, 2015)。但值得注意的是, 现有研究分析所用的数据主要来自 20 世纪八九十 年代(Schaller, 1998; 吴家炎和王伟, 1999)。自21世 纪以来对于白唇鹿的研究进展缓慢, 常见的是基于 以前调查数据的分析预测 (Luo et al, 2015; Wu, 2015), 而该物种在祁连山、昆仑山、唐古拉山等地 


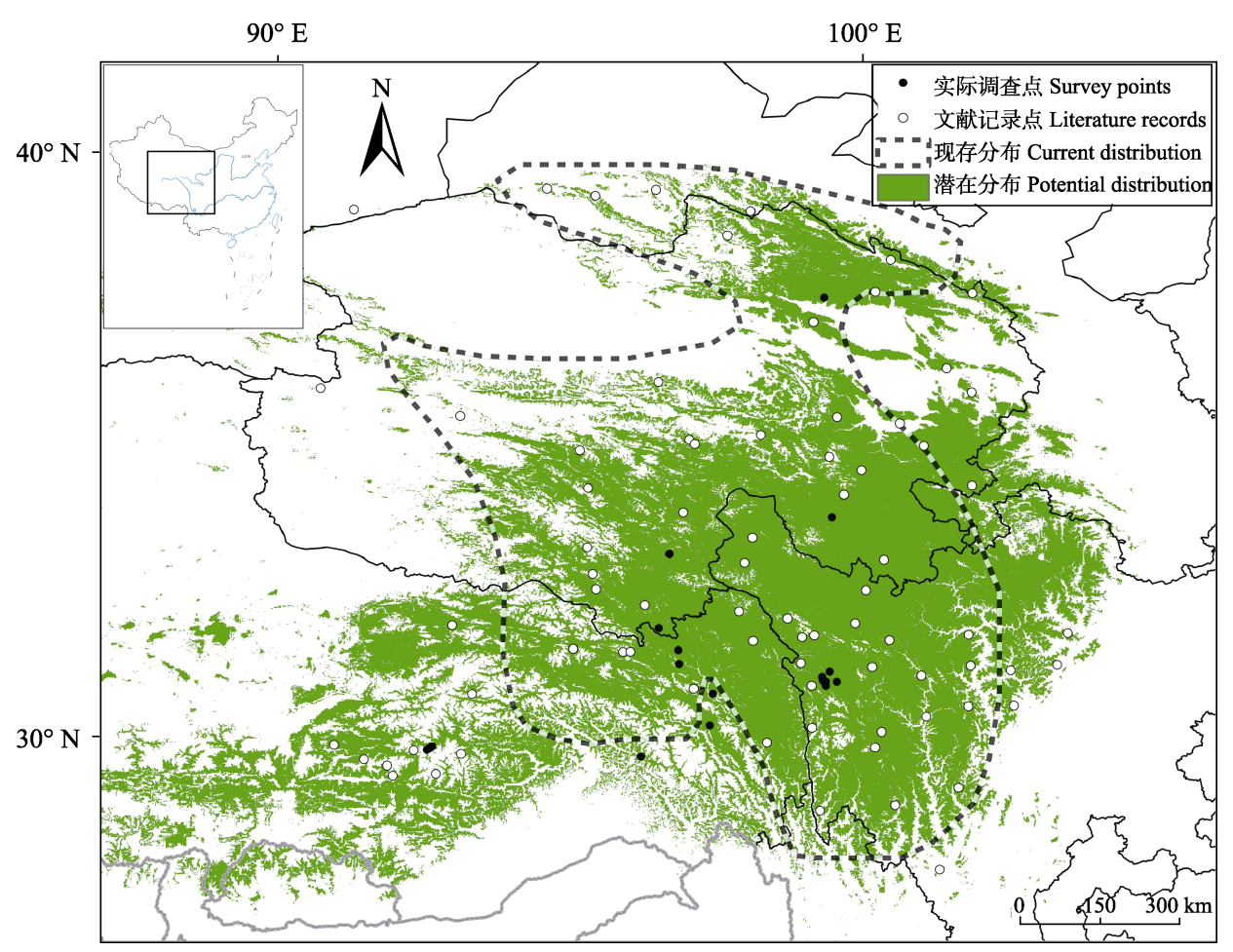

图2 白唇鹿在青藏高原的潜在分布范围

Fig. 2 The potential distribution of white-lipped deer (Przewalskium albirostris) on the Qinghai-Tibetan Plateau

区的分布现状仍不清晰，其谱系地理结构以及迁移 廊道等关键种群信息尚无报道。近些年, 白唇鹿在 青海南部数量有所增加, 在寺庙周边也常常出现 (Leslie, 2010)，同时该物种与家畜和其他有蹄类间 存在竞争(Hughes et al, 2015)。因此, 有必要对白唇 鹿进行深入调查。

我们的刀切法分析结果与Geist (1998)研究结 论一致, 即白唇鹿是适应于高寒环境的青藏高原特 有种, 海拔对其分布范围的影响很大。尽管人类活 动变量 (人类足迹指数和放牧强度)影响相对较小, 但对白唇鹿生境选择的研究中发现白唇鹿回避人 类活动较密集的区域(游章强等, 2014)。与此同时, 夏季NDVI指数可能间接表征了食物资源的丰富程 度, 对白唇鹿的潜在地理分布有决定作用。

近年来, 人们应用物种分布模型预测物种潜在 分布区的研究逐年增多(Engler et al, 2004; Carnaval \& Moritz, 2008), 物种分布模型已经成为生物地理 研究和物种保护的有效工具 (Guisan \& Thuiller, 2005; Escobar et al, 2015)。其中, MaxEnt模型是应用 最广的一种, 自2006年以来已有 1,000 多个相关应 用案例(Merow et al, 2013), 尽管还存在一些缺点
(如对采样偏差敏感), 但该模型只需要物种分布点 数据和相应研究区域的环境信息, 特别是在数据样 本量较少的情况下比其他模型的预测效果更好 (Hernandez et al, 2006; Pearson et al, 2007)。除此之 外, MaxEnt模型可输出连续的结果用以区分环境适 宜性的微小差别, 这在制定保护规划时具有重要价 值(Phillips et al, 2006)。采用野外调查方法确定濒危 物种的所有分布区域需要耗费相当多的人力和物 力, 却依然收效甚微(Rushton et al, 2004)。事实上, 通过模型预测潜在分布区可以提高野外调查的成 功率(Guisan et al, 2006), 也有助于确定珍稀物种的 适宜生境(Pyron et al, 2008), 促进生境评估和保护 工作顺利开展。

\section{参考文献}

Carnaval AC, Moritz C (2008) Historical climate modelling predicts patterns of current biodiversity in the Brazilian Atlantic forest. Journal of Biogeography, 35, 1187-1201.

Engler R, Guisan A, Rechsteiner L (2004) An improved approach for predicting the distribution of rare and endangered species from occurrence and pseudo-absence data. Journal of Applied Ecology, 41, 263-274.

Escobar LE, Awan MN, Qiao HJ (2015) Anthropogenic distur- 
bance and habitat loss for the red-listed Asiatic black bear (Ursus thibetanus): Using ecological niche modeling and nighttime light satellite imagery. Biological Conservation, 191, 400-407.

Fahrig L (2003) Effects of habitat fragmentation on biodiversity. Annual Review of Ecology, Evolution, and Systematics, 34, 487-515.

Geist V (1998) Deer of the World: Their Evolution, Behaviour, and Ecology. Stackpole Books, Mechanicsburg, USA.

Guisan A, Lehmann A, Ferrier S, Austin M, Overton J, Aspinall R, Hastie T (2006) Making better biogeographical predictions of species' distributions. Journal of Applied Ecology, 43, 386-392.

Guisan A, Thuiller W (2005) Predicting species distribution: Offering more than simple habitat models. Ecology Letters, 8, 993-1009.

Harris RB (2015) Cervus albirostris. The IUCN Red List of Threatened Species 2015: e.T4256A61976756. http://www. iucnredlist.org/details/4256/0/. (accessed on 2016-05-02)

Harris RB, Pletscher DH, Loggers CO, Miller DJ (1999) Status and trends of Tibetan plateau mammalian fauna, Yeniugou, China. Biological Conservation, 87, 13-19.

Hernandez PA, Graham CH, Master LL, Albert DL (2006) The effect of sample size and species characteristics on performance of different species distribution modeling methods. Ecography, 29, 773-785.

Hijmans RJ, Cameron SE, Parra JL, Jones PG, Jarvis A (2005) Very high resolution interpolated climate surfaces for global land areas. International Journal of Climatology, 25, $1965-1978$.

Hu JH, Hu HJ, Jiang ZG (2010) The impacts of climate change on the wintering distribution of an endangered migratory bird. Oecologia, 164, 555-565.

Hughes J, Alexander J, Shi K, Riordan P (2015) Confirmation of threatened white-lipped deer (Przewalskium albirostris) in Gansu and Sichuan, China, and their overlap with livestock. Mammalia, 79, 241-244.

Jiang ZG, Jiang JP, Wang YZ, Zhang E, Zhang YY, Li LL, Xie F, Cai B, Cao L, Zheng GM, Dong L, Zhang ZW, Ding P, Luo ZH, Ding CQ, Ma ZJ, Tang SH, Cao WX, Li CW, Hu HJ, Ma Y, Wu Y, Wang YX, Zhou KY, Liu SY, Chen YY, Li JT, Feng ZJ, Wang Y, Wang B, Li C, Song XL, Cai L, Zang CX, Zeng Y, Meng ZB, Fang HX, Ping XG (2016) Red List of China's Vertebrates. Biodiversity Science, 24, 500-551. (in Chinese and in English) [蒋志刚, 江建平, 王 跃招, 张鹗, 张雁云, 李立立, 谢锋, 蔡波, 曹亮, 郑光 美, 董路, 张正旺, 丁平, 罗振华, 丁长青, 马志军, 汤宋 华, 曹文宣, 李春旺, 胡慧建, 马勇, 吴毅, 王应祥, 周开 亚, 刘少英, 陈跃英, 李家堂, 冯祚建, 王燕, 王斌, 李 成, 宋雪琳, 蔡蕾, 藏春釒金, 曾岩, 孟智斌, 方红霞, 平晓 鸽 (2016) 中国脊椎动物红色名录. 生物多样性, 24, 500-551.]

Jiang ZG, Ma KP (2014) The Principles of Conservation Biol- ogy. Science Press, Beijing. (in Chinese) [蒋志刚, 马克平 (2014) 保护生物学原理. 科学出版社, 北京.]

Jiang ZG, Ma Y, Wu Y, Wang YX, Feng ZJ, Zhou KY, Liu SY, Luo ZH, Li CW (2015) Diversity of China's mammals. Biodiversity Science, 23, 351-364. (in Chinese with English abstract) [蒋志刚, 马勇, 吴毅, 王应祥, 冯祚建, 周开亚, 刘少英, 罗振华, 李春旺 (2015) 中国哺乳动物多样性. 生物多样性, 23, 351-364.]

Leslie DM (2010) Przewalskium albirostre (Artiodactyla: Cervidae). Mammalian Species, 42, 7-18.

Liu C, White M, Newell G (2013) Selecting thresholds for the prediction of species occurrence with presence-only data. Journal of Biogeography, 40, 778-789.

Luo ZH, Jiang ZG, Tang SH (2015) Impacts of climate change on distributions and diversity of ungulates on the Tibetan Plateau. Ecological Applications, 25, 24-38.

Merow C, Smith MJ, Silander JA (2013) A practical guide to MaxEnt for modeling species' distributions: What it does, and why inputs and settings matter. Ecography, 36, 1058-1069.

Ohtaishi N, Gao Y (1990) A review of the distribution of all species of deer (Tragulidae, Moschidae and Cervidae) in China. Mammal Review, 20, 125-144.

Pearce JL, Boyce MS (2006) Modelling distribution and abundance with presence-only data. Journal of Applied Ecology, 43, 405-412.

Pearson RG, Raxworthy CJ, Nakamura M, Peterson AT (2007) Predicting species distributions from small numbers of occurrence records: A test case using cryptic geckos in Madagascar. Journal of Biogeography, 34, 102-117.

Phillips SJ, Anderson RP, Schapire RE (2006) Maximum entropy modeling of species geographic distributions. Ecological Modelling, 190, 231-259.

Phillips SJ, Dudík M (2008) Modeling of species distributions with Maxent: New extensions and a comprehensive evaluation. Ecography, 31, 161-175.

Pyron RA, Burbrink FT, Guiher TJ (2008) Claims of potential expansion throughout the US by invasive python species are contradicted by ecological niche models. PLoS ONE, 3, e2931.

Rushton SP, Ormerod SJ, Kerby G (2004) New paradigms for modelling species distributions? Journal of Applied Ecology, 41, 193-200.

Sanderson EW, Jaiteh M, Levy MA, Redford KH, Wannebo AV, Woolmer G (2002) The human footprint and the last of the wild. BioScience, 52, 891-904.

Saupe EE, Qiao HJ, Hendricks JR, Portell RW, Hunter SJ, Soberón J, Lieberman BS (2015) Niche breadth and geographic range size as determinants of species survival on geological time scales. Global Ecology and Biogeography, 24, 1159-1169.

Schaller GB (1998) Wildlife of the Tibetan Steppe. University of Chicago Press, Chicago, Illinois. 
Smith AT, Xie Y (2009) A Guide to the Mammals of China. Hunan Education Press, Changsha. (in Chinese) [Smith AT, 解炎 (2009) 中国兽类野外手册. 湖南教育出版社, 长 沙.]

State Forestry Administration (2009) A Survey on the Resources of Chinese Key Terrestrial Wildlife. China Forestry Publishing House, Beijing. (in Chinese) [国家林业局 (2009) 中国重点陆生野生动物资源调查. 中国林业出版 社, 北京.]

Thorn JS, Nijman V, Smith D, Nekaris KAI (2009) Ecological niche modelling as a technique for assessing threats and setting conservation priorities for Asian slow lorises (Primates: Nycticebus). Diversity and Distributions, 15, 289-298.

USGS (2001) Hydro-1K Elevation Derivative Database. http://edcdaac.usgs.gov/gtopo30/hydro/. (accessed on 201411-26)

Wildlife Conservation (WCS), Center for International Earth Science Information Network (CIESIN) (2005) Last of the Wild Data Version 2, 2005(LTW-2): Global Human Footprint Dataset (Geographic). http://sedac.ciesin.columbia.edu/data/set/wildareas-v2-human-footprint-geographic. (accessed on 2010-11-28)

Wu JG (2015) Detecting and attributing the effect of climate change on the changes in the distribution of Qinghai-Tibet Plateau large mammal species over the past 50 years. Mammal Research, 60, 353-364.

Wu JY, Wang W (1999) The White-lipped Deer of China. China Forestry Publishing House, Beijing. (in Chinese with English abstract) [吴家炎, 王伟 (1999) 中国白唇鹿. 中
国林业出版社, 北京.]

Wu QM, Wang L, Zhu RP, Yang YB, Jin HY, Zou HF (2016) Nesting habitat suitability analysis of red-crowned crane in Zhalong Nature Reserve based on MAXENT modeling. Acta Ecologica Sinica, 36, 3758-3764. (in Chinese with English abstract) [吴庆明, 王磊, 朱瑞萍, 杨宇博, 金洪 阳, 邹红菲 (2016) 基于MAXENT模型的丹顶鹤营巢生 境适宜性分析一以扎龙保护区为例. 生态学报, 36, 3758-3764.]

You ZQ, Tang ZH, Yang YB, Yang LH, Shi HY, Liu H, Gan X, Zheng TC, Jiang ZG (2014) Summer habitat selection by white-lipped deer in the Chaqingsongduo White-lipped Deer National Nature Reserve. Acta Theriologica Sinica, 34, 46-53. (in Chinese with English abstract) [游章强, 唐中海, 杨远斌, 杨丽红, 石红艳, 刘吴, 甘萧, 郑天才, 蒋志刚 (2014) 察青松多白唇鹿国家级自然保护区白唇鹿对夏季 生境的选择. 兽类学报, 34, 46-53.]

Yu D, Chen M, Zhou ZC, Eric R, Tang QG, Liu HZ (2013) Global climate change will severely decrease potential distribution of the East Asian coldwater fish Rhynchocypris oxycephalus (Actinopterygii, Cyprinidae). Hydrobiologia, 700, 23-32.

Zhang RZ (1997) Distribution of Mammalian Species in China. China Forestry Publishing House, Beijing. (in Chinese with English abstract) [张荣祖 (1997) 中国哺乳动物分布. 中 国林业出版社, 北京.]

(责任编委: 蒋学龙 责任编辑: 问文杰)

\section{附录 Supplementary Material}

\section{附录1 所有生物气候变量的主成分分析结果}

Appendix 1 Results of the principal components analysis based on all bioclimatic variables http://www.biodiversity-science.net/fileup/PDF/2017080-1.pdf

\section{附录2 27个初始环境变量之间的相关系数}

Appendix 2 The Pearson correlation coefficients among 27 initial environmental variables http://www.biodiversity-science.net/fileup/PDF/2017080-2.pdf 
崔绍朋, 罗晓, 李春旺, 胡慧建, 蒋志刚. 基于MaxEnt模型预测白唇鹿的潜在分布区. 生物多样性, 2018, 26 (2): 171-176.

http://www.biodiversity-science.net/CN/10.17520/biods.2017080

附录 1 所有生物气候变量的主成分分析结果

Appendix 1 Results of the principal components analysis based on all bioclimatic variables

\begin{tabular}{|c|c|c|}
\hline \multirow{2}{*}{ 变量 Variables } & \multicolumn{2}{|c|}{ 特征向量 Eigenvectors } \\
\hline & PC1 & PC2 \\
\hline 年均温 Annual mean temperature (Bio1) & -0.009 & 0.100 \\
\hline 气温日较差 Mean diurnal range (Bio2) & 0.006 & -0.014 \\
\hline 等温性 Isothermality (Bio3) & -0.002 & -0.002 \\
\hline 气温季节变化 Temperature seasonality (Bio4) & 0.959 & 0.274 \\
\hline 最热月最高气温 Max. temperature of the warmest month (Bio5) & 0.009 & 0.096 \\
\hline 最冷月最低气温 Min. temperature of the coldest month (Bio6) & -0.023 & 0.102 \\
\hline 气温年较差 Temperature of annual range (Bio7) & 0.032 & -0.006 \\
\hline 最湿季均温 Mean temperature of the wettest quarter (Bio8) & 0.003 & 0.100 \\
\hline 最干季均温 Mean temperature of the driest quarter (Bio9) & -0.018 & 0.095 \\
\hline 最暖季均温 Mean temperature of the warmest quarter (Bio10) & 0.004 & 0.100 \\
\hline 最冷季均温. Mean temperature of the coldest quarter (Bio11) & -0.021 & 0.093 \\
\hline 年降水量 Annual precipitation (Bio12) & -0.213 & 0.715 \\
\hline 最湿月降水量 Precipitation of the wettest month (Bio13) & -0.047 & 0.158 \\
\hline 最干月降水量 Precipitation of the driest month (Bio14) & -0.001 & 0.003 \\
\hline 降水季节性变化 Precipitation seasonality (Bio15) & 0.000 & -0.010 \\
\hline 最湿季降水量 Precipitation of the wettest quarter (Bio16) & -0.125 & 0.422 \\
\hline 最干季降水量 Precipitation of the driest quarter (Bio17) & -0.005 & 0.015 \\
\hline 最暖季降水量 Precipitation of the warmest quarter (Bio18) & -0.119 & 0.377 \\
\hline 最冷季降水量 Precipitation of the coldest quarter (Bio19) & -0.005 & 0.018 \\
\hline 贡献率 Contribution rate & $93.98 \%$ & $5.61 \%$ \\
\hline 累积贡献率 Cumulative contribution rate & $93.98 \%$ & $99.59 \%$ \\
\hline
\end{tabular}


附录 227 个初始环境变量之间的相关系数。环境变量含义见表 1 和附录 1 。

Appendix 2 The Pearson correlation coefficients among 27 initial environmental variables. Environmental variable meanings are shown in Table 1 and Appendix 1.

\begin{tabular}{|c|c|c|c|c|c|c|c|c|c|c|c|c|c|c|c|c|c|c|c|c|c|c|c|c|c|c|c|}
\hline & Bio1 & Bio2 & Bio3 & Bio4 & Bio5 & Bio6 & Bio7 & Bio8 & Bio9 & Bio10 & Bio11 & Bio12 & Bio13 & Bio14 & Bio15 & Bio16 & Bio17 & Bio18 & Bio19 & Altitude & HFI & $\begin{array}{l}\text { Stocking } \\
\text { rate }\end{array}$ & Slope & $\begin{array}{l}\text { NDVI_ } \\
\text { Summer }\end{array}$ & $\begin{array}{l}\text { NDVI_ } \\
\text { Winter }\end{array}$ & $\begin{array}{l}\text { NDVI_ } \\
\text { Autumn }\end{array}$ & $\begin{array}{l}\text { NDVI_ } \\
\text { Spring }\end{array}$ \\
\hline Bio1 & 1 & & & & & & & & & & & & & & & & & & & & & & & & & & \\
\hline Bio2 & -0.57 & 1 & & & & & & & & & & & & & & & & & & & & & & & & & \\
\hline Bio3 & -0.06 & 0.04 & 1 & & & & & & & & & & & & & & & & & & & & & & & & \\
\hline Bio4 & -0.20 & 0.58 & -0.76 & 1 & & & & & & & & & & & & & & & & & & & & & & & \\
\hline Bio5 & 0.86 & -0.22 & -0.44 & 0.33 & 1 & & & & & & & & & & & & & & & & & & & & & & \\
\hline Bio6 & 0.94 & -0.76 & 0.14 & -0.51 & 0.63 & 1 & & & & & & & & & & & & & & & & & & & & & \\
\hline Bio7 & -0.32 & 0.74 & -0.62 & 0.97 & 0.21 & -0.63 & 1 & & & & & & & & & & & & & & & & & & & & \\
\hline Bio8 & 0.93 & -0.36 & -0.33 & 0.16 & 0.98 & 0.76 & 0.03 & 1 & & & & & & & & & & & & & & & & & & & \\
\hline Bio9 & 0.94 & -0.62 & 0.18 & -0.44 & 0.68 & 0.97 & -0.53 & 0.79 & 1 & & & & & & & & & & & & & & & & & & \\
\hline Bio10 & 0.93 & -0.37 & -0.34 & 0.16 & 0.98 & 0.76 & 0.03 & 1.00 & 0.79 & 1 & & & & & & & & & & & & & & & & & \\
\hline Bio11 & 0.94 & -0.69 & 0.21 & -0.52 & 0.63 & 0.99 & -0.62 & 0.76 & 0.98 & 0.76 & 1 & & & & & & & & & & & & & & & & \\
\hline Bio12 & 0.63 & -0.70 & 0.42 & -0.73 & 0.22 & 0.79 & -0.78 & 0.38 & 0.75 & 0.37 & 0.80 & 1 & & & & & & & & & & & & & & & \\
\hline Bio13 & 0.60 & -0.67 & 0.44 & -0.72 & 0.19 & 0.76 & -0.77 & 0.35 & 0.73 & 0.34 & 0.77 & 0.99 & 1 & & & & & & & & & & & & & & \\
\hline Bio14 & 0.60 & -0.81 & 0.10 & -0.58 & 0.26 & 0.75 & -0.69 & 0.40 & 0.67 & 0.39 & 0.72 & 0.76 & 0.69 & 1 & & & & & & & & & & & & & \\
\hline Bio15 & -0.51 & 0.42 & 0.24 & 0.05 & -0.46 & -0.47 & 0.13 & -0.50 & -0.42 & -0.49 & -0.45 & -0.30 & -0.21 & -0.51 & 1 & & & & & & & & & & & & \\
\hline Bio16 & 0.61 & -0.67 & 0.44 & -0.72 & 0.19 & 0.76 & -0.77 & 0.35 & 0.73 & 0.34 & 0.78 & 0.99 & 1.00 & 0.71 & -0.23 & 1 & & & & & & & & & & & \\
\hline Bio17 & 0.63 & -0.80 & 0.21 & -0.64 & 0.26 & 0.79 & -0.73 & 0.41 & 0.73 & 0.40 & 0.77 & 0.85 & 0.79 & 0.97 & -0.48 & 0.81 & 1 & & & & & & & & & & \\
\hline Bio18 & 0.60 & -0.68 & 0.46 & -0.74 & 0.18 & 0.76 & -0.79 & 0.33 & 0.73 & 0.33 & 0.78 & 0.99 & 0.99 & 0.72 & -0.24 & 0.99 & 0.82 & 1 & & & & & & & & & \\
\hline Bio19 & 0.61 & -0.74 & 0.22 & -0.61 & 0.25 & 0.76 & -0.70 & 0.40 & 0.70 & 0.39 & 0.74 & 0.85 & 0.79 & 0.94 & -0.44 & 0.81 & 0.98 & 0.82 & 1 & & & & & & & & \\
\hline Altitude & -0.90 & 0.40 & 0.42 & -0.19 & -0.97 & -0.73 & -0.05 & -0.98 & -0.75 & -0.98 & -0.72 & -0.38 & -0.34 & -0.41 & 0.56 & -0.34 & -0.42 & -0.33 & -0.40 & 1 & & & & & & & \\
\hline HFI & 0.51 & -0.58 & 0.01 & -0.39 & 0.28 & 0.59 & -0.46 & 0.37 & 0.51 & 0.37 & 0.58 & 0.46 & 0.43 & 0.48 & -0.39 & 0.43 & 0.47 & 0.43 & 0.42 & -0.40 & 1 & & & & & & \\
\hline Stocking rate & 0.25 & -0.29 & -0.03 & -0.17 & 0.15 & 0.28 & -0.20 & 0.18 & 0.24 & 0.19 & 0.27 & 0.18 & 0.17 & 0.20 & -0.17 & 0.17 & 0.18 & 0.17 & 0.15 & -0.21 & 0.38 & 1 & & & & & \\
\hline Slope & 0.02 & -0.20 & 0.46 & -0.49 & -0.23 & 0.17 & -0.45 & -0.15 & 0.15 & -0.16 & 0.19 & 0.25 & 0.23 & 0.18 & -0.08 & 0.24 & 0.21 & 0.27 & 0.20 & 0.19 & 0.10 & 0.02 & 1 & & & & \\
\hline NDVI_Summer & 0.40 & -0.61 & 0.38 & -0.73 & 0.00 & 0.59 & -0.74 & 0.14 & 0.51 & 0.13 & 0.59 & 0.67 & 0.63 & 0.63 & -0.40 & 0.64 & 0.65 & 0.66 & 0.61 & -0.14 & 0.55 & 0.25 & 0.39 & 1 & & & \\
\hline NDVI_Winter & 0.64 & -0.69 & 0.38 & -0.69 & 0.24 & 0.79 & -0.76 & 0.39 & 0.74 & 0.39 & 0.79 & 0.80 & 0.77 & 0.72 & -0.36 & 0.79 & 0.77 & 0.80 & 0.75 & -0.38 & 0.45 & 0.20 & 0.40 & 0.82 & 1 & & \\
\hline NDVI_Autumn & 0.52 & -0.66 & 0.44 & -0.77 & 0.09 & 0.70 & -0.80 & 0.24 & 0.64 & 0.24 & 0.71 & 0.76 & 0.72 & 0.69 & -0.41 & 0.74 & 0.73 & 0.76 & 0.69 & -0.24 & 0.54 & 0.24 & 0.43 & 0.96 & 0.92 & 1 & \\
\hline NDVI_Spring & 0.62 & -0.71 & 0.32 & -0.68 & 0.23 & 0.77 & -0.74 & 0.38 & 0.71 & 0.37 & 0.77 & 0.79 & 0.75 & 0.72 & -0.40 & 0.77 & 0.77 & 0.78 & 0.74 & -0.38 & 0.51 & 0.23 & 0.38 & 0.87 & 0.98 & 0.94 & 1 \\
\hline
\end{tabular}

